\title{
The split variational inequality problem and its algorithm iteration
}

\author{
Yonghong Yao ${ }^{a}$, Xiaoxue Zheng ${ }^{a}$, Limin Leng ${ }^{a}$, Shin Min Kang ${ }^{b, c, *}$ \\ a Department of Mathematics, Tianjin Polytechnic University, Tianjin 300387, China. \\ ${ }^{b}$ Center for General Education, China Medical University, Taichung 40402, Taiwan. \\ ${ }^{c}$ Department of Mathematics and the RINS, Gyeongsang National University, Jinju 52828, Korea.
}

Communicated by $\mathrm{X}$. Qin

\begin{abstract}
The split variational inequality problem under a nonlinear transformation has been considered. An iterative algorithm is presented to solve this split problem. Strong convergence results are obtained. (C)2017 All rights reserved.
\end{abstract}

Keywords: Split variational inequality, iterative algorithm, nonlinear transformation.

2010 MSC: 49J53, 49M37, 65K10, 90C25.

\section{Introduction}

Let $\mathcal{H}$ be a real Hilbert space with its inner product $\langle\cdot, \cdot\rangle$ and norm $\|\cdot\|$. Let $\mathcal{C} \subset \mathcal{H}$ be a nonempty closed convex set. Let $\mathcal{A}: \mathcal{C} \rightarrow \mathcal{H}$ and $\psi: \mathcal{C} \rightarrow \mathcal{C}$ be three nonlinear operators. Now we consider the following variational inequality of finding $u \in \mathcal{C}$ such that

$$
\langle\mathcal{A u}, \psi(v)-\psi(\mathfrak{u})\rangle \geqslant 0, \quad \forall v \in \mathcal{C} .
$$

Denote the set of the solutions of $(1.1)$ by $\operatorname{VI}(\mathcal{A}, \psi, \mathcal{C})$.

If $\psi \equiv \mathrm{I}$, then (1.1) reduces to the variational inequality of finding $u \in \mathcal{C}$ such that

$$
\langle\mathcal{A u}, v-u\rangle \geqslant 0, \quad \forall v \in \mathcal{C} .
$$

Denote the set of the solutions of (1.2) by $\operatorname{VI}(\mathcal{A}, \mathcal{C})$.

Variational inequalities have played a critical and significant part in the study of several unrelated problems arising in physics, finance, economics, network analysis, elasticity, optimization, water resources, medical images and structural analysis. For some related work, please refer to Ceng et al. [5, 6], Cianciaruso et al. [12], Glowinski [13], Iusem [16], Korpelevič [17], Noor [1], Qin and Cho [19], Qin and Yao [20], Yao et al. [27, 29, 31, 32], Zegeye et al. [34], and Zhang et al. [35].

\footnotetext{
*Corresponding author

Email addresses: yaoyonghong@aliyun.com (Yonghong Yao), zhengxiaoxue1991@aliyun.com (Xiaoxue Zheng), lenglimin@aliyun.com (Limin Leng), smkang@gnu.ac.kr (Shin Min Kang)

doi:10.22436/jnsa.010.05.31
} 
Recently, the split feasibility problem has been studied extensively, see [2-4, 7-9, 24-26, 28, 33]. The split feasibility problem is formulated as finding $\hat{v}$ such that

$$
\hat{v} \in \mathcal{C} \text { and } \psi(\hat{v}) \in Q,
$$

where $\mathcal{C}$ and $Q$ are two closed convex subsets of two Hilbert spaces $\mathcal{H}_{1}$ and $\mathcal{H}_{2}$, respectively, and $\psi$ : $\mathcal{H}_{1} \rightarrow \mathcal{H}_{2}$ is a bounded linear operator. Such problems arise in the field of intensity-modulated radiation therapy.

\section{Special cases.}

(i) If $\mathcal{C}=\operatorname{Fix}(S)$ and $Q=\operatorname{Fix}(T)$, then (1.3) reduces to the split common fixed point problem which was first introduced by Censor and Segal [11]. The reader can refer to He and Du [14,15] and Yao et al. [30].

(ii) If $\mathcal{C}=\operatorname{VI}(\mathcal{A}, \mathcal{D})$ and $\mathcal{Q}=\operatorname{VI}(\mathcal{B}, \mathcal{E})$, then (1.3) reduces to the split variational inequality problem which was studied in [10].

In the present manuscript, we focus on the following split variational inequality problem of finding a point $\hat{v}$ such that

$$
\hat{v} \in \operatorname{VI}(\mathcal{A}, \psi, \mathcal{C}) \text { and } \psi(\hat{v}) \in \operatorname{VI}(\mathcal{B}, \mathcal{C}) .
$$

Remark 1.1. In the existing literature, the split problem that requires to find a point of an operator in one space whose image under a linear transformation is a point of another operator in the image space. However, in (1.4), the transformation $\psi$ is nonlinear.

In order to solve (1.4), we introduce a new iterative algorithm. Under some mild assumptions, we show the strong convergence of the presented algorithm.

\section{Preliminaries}

Let $\mathcal{C}$ be a nonempty closed convex subset of a real Hilbert space $\mathcal{H}$.

Definition 2.1. An operator $\vartheta: \mathcal{C} \rightarrow \mathcal{C}$ is said to be L-Lipschitz continuous if there exists a constant $L>0$ such that

$$
\left\|\vartheta(u)-\vartheta\left(u^{\dagger}\right)\right\| \leqslant L\left\|u-u^{\dagger}\right\|
$$

for all $u, u^{\dagger} \in \mathcal{C}$.

Definition 2.2. An operator $\mathcal{A}: \mathcal{C} \rightarrow \mathcal{H}$ is said to be

(1) monotone if

$$
\left\langle\mathcal{A u}-\mathcal{A} u^{\dagger}, \mathrm{u}-\mathrm{u}^{\dagger}\right\rangle \geqslant 0, \quad \forall \mathrm{u}, \mathrm{u}^{\dagger} \in \mathcal{C} ;
$$

(2) strongly monotone if there exists a constant $\delta>0$ such that

$$
\left\langle\mathcal{A} u-\mathcal{A} u^{\dagger}, u-u^{\dagger}\right\rangle \geqslant \delta\left\|u-u^{\dagger}\right\|^{2}, \quad \forall u, u^{\dagger} \in \mathcal{C} ;
$$

(3) inverse strongly monotone if there exists $\eta>0$ such that

$$
\left\langle\mathcal{A} u-\mathcal{A} u^{\dagger}, u-u^{\dagger}\right\rangle \geqslant \eta\left\|\mathcal{A} u-\mathcal{A} u^{\dagger}\right\|^{2}, \quad \forall u, u^{\dagger} \in \mathcal{C} .
$$

Let $\psi: \mathcal{C} \rightarrow \mathcal{C}$ be a nonlinear operator.

Definition 2.3. An operator $\mathcal{A}: \mathcal{C} \rightarrow \mathcal{H}$ is said to be $\eta$-inverse strongly $\psi$-monotone iff

$$
\left\langle\mathcal{A} u-\mathcal{A} u^{\dagger}, \psi(u)-\psi\left(u^{\dagger}\right)\right\rangle \geqslant \eta\left\|\mathcal{A} u-\mathcal{A} u^{\dagger}\right\|^{2}
$$

for all $u, u^{\dagger} \in \mathcal{C}$ and for some $\eta>0$. 
Let $\mathcal{A}: \mathcal{H} \rightarrow 2^{\mathcal{H}}$ be a set-valued operator. The effective domain of $\mathcal{A}$ is denoted by $\operatorname{dom}(\mathcal{A})$, that is, $\operatorname{dom}(\mathcal{A})=\{\mathrm{x} \in \mathrm{H}: \mathcal{A x} \neq \emptyset\}$.

Definition 2.4. A set-valued operator $\mathcal{A}$ is said to be monotone on $\mathcal{H}$ iff

$$
\langle x-y, u-v\rangle \geqslant 0
$$

for all $x, y \in \operatorname{dom}(\mathcal{A}), u \in \mathcal{A x}$ and $v \in \mathcal{A y}$.

A monotone operator $\mathcal{A}$ on $\mathcal{H}$ is said to be maximal iff its graph is not strictly contained in the graph of any other monotone operator on $\mathcal{H}$.

For every point $u \in \mathcal{H}$, there exists a unique nearest point in $\mathcal{C}$, denoted by proj $j_{\mathcal{C}}[u]$ such that

$$
\left\|u-\operatorname{proj}_{\mathfrak{C}}[u]\right\| \leqslant\left\|u-u^{\dagger}\right\|
$$

for all $\mathrm{u}^{\dagger} \in \mathcal{C}$.

The mapping proj $_{\mathcal{C}}$ is called the metric projection of $\mathcal{H}$ onto $\mathcal{C}$. It is well-known that proj $\mathfrak{C}_{\mathcal{C}}$ is a typical firmly nonexpansive mapping and is characterized by the following property

$$
\left\langle u-\operatorname{proj}_{\mathfrak{C}}[u], u^{\dagger}-\operatorname{proj}_{\mathfrak{C}}[u]\right\rangle \leqslant 0, \quad \forall u \in \mathcal{H}, u^{\dagger} \in \mathcal{C} .
$$

Lemma 2.5 ([22]). Let $\mathcal{C}$ be a nonempty closed convex subset of a real Hilbert space $\mathcal{H}$ and $\mathcal{A}: \mathcal{C} \rightarrow \mathcal{H}$ be a छ-inverse strongly monotone mapping. Then,

$$
\left\|(\mathrm{I}-\delta \mathcal{A}) \mathrm{u}-(\mathrm{I}-\delta \mathcal{A}) \mathrm{u}^{\dagger}\right\|^{2} \leqslant\|x-y\|^{2}+\delta(\delta-2 \xi)\left\|\mathcal{A} u-\mathcal{A} u^{\dagger}\right\|^{2}, \quad \forall \mathrm{u}, \mathrm{u}^{\dagger} \in \mathcal{C},
$$

where $\delta>0$ is a constant.

Lemma 2.6 ([23]). Let $\left\{\varpi_{n}\right\} \subset[0, \infty),\left\{\mu_{n}\right\} \subset(0,1)$, and $\left\{\rho_{n}\right\}$ be three sequences such that

$$
\varpi_{n+1} \leqslant\left(1-\mu_{n}\right) \varpi_{n}+\rho_{n}, \quad \forall n \geqslant 1 .
$$

Assume the following restrictions are satisfied

(i) $\sum_{n=1}^{\infty} \mu_{n}=\infty$;

(ii) $\varlimsup_{n \rightarrow \infty} \frac{\rho_{n}}{\mu_{n}} \leqslant 0$ or $\sum_{n=1}^{\infty}\left|\rho_{n}\right|<\infty$.

Then $\lim _{n \rightarrow \infty}{\varpi_{n}}=0$.

Lemma 2.7 ([18]). Let $\left\{w_{n}\right\}$ be a sequence of real numbers. Assume $\left\{w_{n}\right\}$ does not decrease at infinity, that is, there exists at least a subsequence $\left\{w_{n_{k}}\right\}$ of $\left\{w_{n}\right\}$ such that $w_{n_{k}} \leqslant w_{n_{k}+1}$ for all $k \geqslant 0$. For every $n \geqslant N_{0}$, define an integer sequence $\{\tau(\mathrm{n})\}$ as

$$
\tau(n)=\max \left\{i \leqslant n: w_{n_{i}}<w_{n_{i}+1}\right\} .
$$

Then $\tau(\mathrm{n}) \rightarrow \infty$ as $\mathrm{n} \rightarrow \infty$ and for all $\mathrm{n} \geqslant \mathrm{N}_{0}$

$$
\max \left\{w_{\tau(\mathfrak{n})}, w_{n}\right\} \leqslant w_{\tau(\mathfrak{n})+1} .
$$

\section{Main results}

In this section, we study the split variational inequality problem and the convergence analysis of its iterative algorithm.

Let $\mathcal{H}$ be a real Hilbert space and $\mathcal{C} \subset \mathcal{H}$ be a nonempty closed convex set. Let $\psi: \mathcal{C} \rightarrow \mathcal{C}$ be a weakly continuous and $\delta$-strongly monotone mapping such that its range $\mathrm{R}(\psi)=\mathcal{C}$. Let $\mathcal{A}: \mathcal{C} \rightarrow \mathcal{H}$ be an $\eta$-inverse strongly $\psi$-monotone mapping. Let $\mathcal{B}: \mathcal{C} \rightarrow \mathcal{H}$ be a $\xi$-inverse strongly monotone mapping. 
Remark 3.1. For $u, v \in \mathcal{C}$, since $\psi$ is $\delta$-strongly monotone, we get

$$
\delta\|u-v\|^{2} \leqslant\langle\psi(u)-\psi(v), u-v\rangle \leqslant\|\psi(u)-\psi(v)\|\|u-v\| .
$$

Then,

$$
\|\psi(u)-\psi(v)\| \geqslant \delta\|u-v\|, \quad \forall u, v \in \mathcal{C} .
$$

In view of the assumptions on the operator $\psi$, we know $\psi^{-1}$ is single-valued and Lipschitz continuous.

Problem 3.2. The split variational inequality problem is to find $\hat{u}$ such that

$$
\hat{\mathrm{u}} \in \operatorname{VI}(\mathcal{A}, \psi, \mathcal{C}) \text { and } \quad \psi(\hat{\mathrm{u}}) \in \mathrm{VI}(\mathcal{B}, \mathcal{C}) \text {. }
$$

Denote the set of solutions of (3.2) by $\Lambda$, i.e., $\Lambda=\operatorname{VI}(\mathcal{A}, \psi, \mathcal{C}) \cap \psi^{-1}(\operatorname{VI}(\mathcal{B}, \mathcal{C}))$. Throughout, we assume $\Lambda \neq \emptyset$.

Algorithm 3.3. For given initial value $x_{0} \in \mathcal{C}$, define the sequence $\left\{x_{n}\right\}$ by the following form

$$
\left\{\begin{array}{l}
u_{n}=\operatorname{proj}_{\mathcal{e}}\left[\psi\left(x_{n}\right)-\zeta_{n} \mathcal{A} x_{n}\right], \\
v_{n}=\operatorname{proj}_{\mathcal{C}}\left[\eta_{n} \mu \vartheta\left(x_{n}\right)+\left(1-\eta_{n}\right)\left(u_{n}-\delta_{n} \mathcal{B} u_{n}\right)\right], \\
\psi\left(x_{n+1}\right)=\xi_{n} \psi\left(x_{n}\right)+\left(1-\xi_{n}\right) v_{n}, \quad n \geqslant 0,
\end{array}\right.
$$

where $\vartheta: \mathcal{C} \rightarrow \mathcal{H}$ is an L-Lipschitz continuous mapping, $\left\{\eta_{n}\right\},\left\{\xi_{n}\right\}$, and $\left\{\delta_{n}\right\}$ are three real number sequences in $[0,1],\left\{\zeta_{n}\right\}$ is a real number sequence in $(0, \infty)$, and $0<\mu<\delta / L$ is a constant.

Remark 3.4. The solution of the variational inequality of finding $x^{*} \in \Lambda$ such that

$$
\left\langle\mu \vartheta\left(x^{*}\right)-\psi\left(x^{*}\right), \psi(x)-\psi\left(x^{*}\right)\right\rangle \leqslant 0, \quad \forall x \in \Lambda
$$

is unique. As a matter of fact, assume that both $x^{*}$ and $\tilde{x}$ solve (3.4). Then,

$$
\left\langle\mu \vartheta\left(x^{*}\right)-\psi\left(x^{*}\right), \psi(\tilde{x})-\psi\left(x^{*}\right)\right\rangle \leqslant 0 \text { and }\left\langle\mu \vartheta(\tilde{x})-\psi(\tilde{x}), \psi\left(x^{*}\right)-\psi(\tilde{x})\right\rangle \leqslant 0 .
$$

Adding up the above two inequalities, we deduce

$$
\left\langle\mu \vartheta(\tilde{x})-\psi(\tilde{x})-\mu \vartheta\left(x^{*}\right)+\psi\left(x^{*}\right), \psi\left(x^{*}\right)-\psi(\tilde{x})\right\rangle \leqslant 0 .
$$

It follows that

$$
\left\|\psi\left(x^{*}\right)-\psi(\tilde{x})\right\|^{2} \leqslant \mu\left\langle\vartheta\left(x^{*}\right)-\vartheta(\tilde{x}), \psi\left(x^{*}\right)-\psi(\tilde{x})\right\rangle \leqslant \mu\left\|\vartheta\left(x^{*}\right)-\vartheta(\tilde{x})\right\|\left\|\psi\left(x^{*}\right)-\psi(\tilde{x})\right\| .
$$

This together with (3.1) implies that

$$
\delta\left\|x^{*}-\tilde{x}\right\| \leqslant\left\|\psi\left(x^{*}\right)-\psi(\tilde{x})\right\| \leqslant \mu\left\|\vartheta\left(x^{*}\right)-\vartheta(\tilde{x})\right\| \leqslant \mu L\left\|x^{*}-\tilde{x}\right\| .
$$

We get $x^{*}=\tilde{x}$ immediately because of $\mu \mathrm{L}<\delta$. So, the variational inequality (3.4) has a unique solution denoted by $\hat{u}$.

Proposition 3.5. Let $\mathcal{C}$ be a nonempty closed convex subset of a real Hilbert space $\mathcal{H}$. Let $\mathcal{A}: \mathcal{C} \rightarrow \mathcal{H}$ be an $\eta$-inverse strongly $\psi$-monotone mapping. Then,

$$
\|(\psi(x)-\zeta \mathcal{A} x)-(\psi(y)-\zeta \mathcal{A} y)\|^{2} \leqslant\|\psi(x)-\psi(y)\|^{2}+\zeta(\zeta-2 \eta)\|\mathcal{A} x-\mathcal{A} y\|^{2}, \quad \forall x, y \in \mathcal{C} .
$$

Proof. In fact,

$$
\begin{aligned}
\|(\psi(x)-\zeta \mathcal{A} x)-(\psi(y)-\zeta \mathcal{A} y)\|^{2} & =\|\psi(x)-\psi(y)\|^{2}-2 \zeta\langle\mathcal{A} x-\mathcal{A} y, \psi(x)-\psi(y)\rangle+\zeta^{2}\|\mathcal{A} x-\mathcal{A} y\|^{2} \\
& \leqslant\|\psi(x)-\psi(y)\|^{2}-2 \zeta \eta\|\mathcal{A} x-\mathcal{A} y\|^{2}+\zeta^{2}\|\mathcal{A} x-\mathcal{A} y\|^{2} \\
& \leqslant\|\psi(x)-\psi(y)\|^{2}+\zeta(\zeta-2 \eta)\|\mathcal{A} x-\mathcal{A} y\|^{2}
\end{aligned}
$$


Theorem 3.6. Assume the following conditions are satisfied:

(C1): $\lim _{n \rightarrow \infty} \eta_{n}=0$ and $\sum_{n} \eta_{n}=\infty$;

(C2): $0<\underline{\lim }_{n \rightarrow \infty} \xi_{n} \leqslant \varlimsup_{\lim _{n \rightarrow \infty}} \xi_{n}<1$ and $\delta \in(L \mu, 2 \eta)$;

(C3): $0<\underline{\lim }_{n \rightarrow \infty} \zeta_{n} \leqslant \varlimsup_{n \rightarrow \infty} \zeta_{n}<2 \eta$ and $0<\underline{\lim }_{n \rightarrow \infty} \delta_{n} \leqslant \varlimsup_{n \rightarrow \infty} \delta_{n}<2 \xi$.

Then the sequence $\left\{x_{n}\right\}$ generated by (3.3) converges strongly to $\hat{u} \in \Lambda$ which solves the variational inequality (3.4).

Proof. Note that $\hat{u} \in \operatorname{VI}(\mathcal{A}, \psi, \mathcal{C})$ and $\psi(\hat{u}) \in \mathrm{VI}(\mathcal{B}, \mathcal{C})$. Using $(2.1)$, we deduce $\psi(\hat{u})=\operatorname{proj}_{\mathcal{C}}[\psi(\hat{u})-$ $\left.\zeta_{n} \mathcal{A} \hat{u}\right]=\operatorname{proj}_{\mathcal{C}}\left[\psi(\hat{u})-\delta_{n} \mathcal{B} \psi(\hat{u})\right]$ for all $n \geqslant 0$. First, from (2.2), we have

$$
\begin{aligned}
\left\|\left(u_{n}-\delta_{n} \mathcal{B} u_{n}\right)-\left(\psi(\hat{u})-\delta_{n} \mathcal{B} \psi(\hat{u})\right)\right\|^{2} & \leqslant\left\|u_{n}-\psi(\hat{u})\right\|^{2}+\delta_{n}\left(\delta_{n}-2 \xi\right)\left\|\mathcal{B} u_{n}-\mathcal{B} \psi(\hat{u})\right\|^{2} \\
& \leqslant\left\|u_{n}-\psi(\hat{u})\right\|^{2} .
\end{aligned}
$$

By (3.5), we get

$$
\begin{aligned}
\left\|u_{n}-\psi(\hat{u})\right\|^{2} & =\left\|\operatorname{proj}_{\mathcal{C}}\left[\psi\left(x_{n}\right)-\zeta_{n} \mathcal{A} x_{n}\right]-\operatorname{proj}_{\mathcal{C}}\left[\psi(\hat{u})-\zeta_{n} \mathcal{A} \hat{u}\right]\right\|^{2} \\
& \leqslant\left\|\left(\psi\left(x_{n}\right)-\zeta_{n} \mathcal{A} x_{n}\right)-\left(\psi(\hat{u})-\zeta_{n} \mathcal{A} \hat{u}\right)\right\|^{2} \\
& \leqslant\left\|\psi\left(x_{n}\right)-\psi(\hat{u})\right\|^{2}+\zeta_{n}\left(\zeta_{n}-2 \eta\right)\left\|\mathcal{A} x_{n}-\mathcal{A} \hat{u}\right\|^{2} \\
& \leqslant\left\|\psi\left(x_{n}\right)-\psi(\hat{u})\right\|^{2}
\end{aligned}
$$

and

$$
\begin{aligned}
& \left\|\psi\left(x_{n+1}\right)-\zeta_{n+1} \mathcal{A} x_{n+1}-\left(\psi\left(x_{n}\right)-\zeta_{n+1} \mathcal{A} x_{n}\right)\right\|^{2} \\
& \quad \leqslant\left\|\psi\left(x_{n+1}\right)-\psi\left(x_{n}\right)\right\|^{2}+\zeta_{n+1}\left(\zeta_{n+1}-2 \eta\right)\left\|\mathcal{A} x_{n+1}-\mathcal{A} x_{n}\right\|^{2} .
\end{aligned}
$$

From (3.1), (3.3), (3.6), and (3.7), we have

$$
\begin{aligned}
\left\|v_{n}-\psi(\hat{u})\right\|= & \left\|\operatorname{proj}_{\mathcal{C}}\left[\eta_{n} \mu \vartheta\left(x_{n}\right)+\left(1-\eta_{n}\right)\left(u_{n}-\delta_{n} \mathcal{B} u_{n}\right)\right]-\operatorname{proj}_{\mathcal{C}}\left[\psi(\hat{u})-\delta_{n} \mathcal{B} \psi(\hat{u})\right]\right\| \\
\leqslant & \left\|\eta_{n}\left(\mu \vartheta\left(x_{n}\right)-\psi(\hat{u})+\delta_{n} \mathcal{B} \psi(\hat{u})\right)+\left(1-\eta_{n}\right)\left(\left(u_{n}-\delta_{n} \mathcal{B} u_{n}\right)-\left(\psi(\hat{u})-\delta_{n} \mathcal{B} \psi(\hat{u})\right)\right)\right\| \\
\leqslant & \eta_{n}\left\|\mu \vartheta\left(x_{n}\right)-\mu \vartheta(\hat{u})\right\|+\eta_{n}\left\|\mu \vartheta(\hat{u})-\psi(\hat{u})+\delta_{n} \mathcal{B} \psi(\hat{u})\right\| \\
& +\left(1-\eta_{n}\right)\left\|\left(u_{n}-\delta_{n} \mathcal{B} u_{n}\right)-\left(\psi(\hat{u})-\delta_{n} \mathcal{B} \psi(\hat{u})\right)\right\| \\
\leqslant & \eta_{n} \mu L\left\|x_{n}-\hat{u}\right\|+\eta_{n}\left\|\mu \vartheta(\hat{u})-\psi(\hat{u})+\delta_{n} \mathcal{B} \psi(\hat{u})\right\|+\left(1-\eta_{n}\right)\left\|u_{n}-\psi(\hat{u})\right\| \\
\leqslant & \eta_{n} \mu L / \delta\left\|\psi\left(x_{n}\right)-\psi(\hat{u})\right\|+\eta_{n}\left\|\mu \vartheta(\hat{u})-\psi(\hat{u})+\delta_{n} \mathcal{B} \psi(\hat{u})\right\|+\left(1-\eta_{n}\right)\left\|\psi\left(x_{n}\right)-\psi(\hat{u})\right\| \\
= & {\left[1-(1-\mu L / \delta) \eta_{n}\right]\left\|\psi\left(x_{n}\right)-\psi(\hat{u})\right\|+\eta_{n}\left\|\mu \vartheta(\hat{u})-\psi(\hat{u})+\delta_{n} \mathcal{B} \psi(\hat{u})\right\| } \\
\leqslant & {\left[1-(1-\mu L / \delta) \eta_{n}\right]\left\|\psi\left(x_{n}\right)-\psi(\hat{u})\right\|+\eta_{n}(\|\mu \vartheta(\hat{u})-\psi(\hat{u})\|+2 \xi\|\mathcal{B} \psi(\hat{u})\|) . }
\end{aligned}
$$

By combination of (3.6), (3.7), and (3.8), we obtain

$$
\begin{aligned}
& \left\|v_{n}-\psi(\hat{u})\right\|^{2} \\
& \leqslant\left\|\eta_{n}\left(\mu \vartheta\left(x_{n}\right)-\psi(\hat{u})+\delta_{n} \mathcal{B} \psi(\hat{u})\right)+\left(1-\eta_{n}\right)\left(\left(u_{n}-\delta_{n} \mathcal{B} u_{n}\right)-\left(\psi(\hat{u})-\delta_{n} \mathcal{B} \psi(\hat{u})\right)\right)\right\|^{2} \\
& \leqslant \eta_{n}\left\|\mu \vartheta\left(x_{n}\right)-\psi(\hat{u})+\delta_{n} \mathcal{B} \psi(\hat{u})\right\|^{2}+\left(1-\eta_{n}\right)\left\|\left(u_{n}-\delta_{n} \mathcal{B} u_{n}\right)-\left(\psi(\hat{u})-\delta_{n} \mathcal{B} \psi(\hat{u})\right)\right\|^{2} \\
& \leqslant \eta_{n}\left\|\mu \vartheta\left(x_{n}\right)-\psi(\hat{u})+\delta_{n} \mathcal{B} \psi(\hat{u})\right\|^{2}+\left(1-\eta_{n}\right)\left[\left\|u_{n}-\psi(\hat{u})\right\|^{2}\right. \\
& \left.+\delta_{n}\left(\delta_{n}-2 \xi\right)\left\|\mathcal{B} u_{n}-\mathcal{B} \psi(\hat{u})\right\|^{2}\right] \\
& \leqslant \eta_{n}\left\|\mu \vartheta\left(x_{n}\right)-\psi(\hat{u})+\delta_{n} \mathcal{B} \psi(\hat{u})\right\|^{2}+\left(1-\eta_{n}\right)\left[\left\|\psi\left(x_{n}\right)-\psi(\hat{u})\right\|^{2}\right. \\
& \left.+\delta_{n}\left(\delta_{n}-2 \xi\right)\left\|\mathcal{B} u_{n}-\mathcal{B} \psi(\hat{u})\right\|^{2}+\zeta_{n}\left(\zeta_{n}-2 \eta\right)\left\|\mathcal{A} x_{n}-\mathcal{A} \hat{u}\right\|^{2}\right] \\
& \leqslant \eta_{n}\left\|\mu \vartheta\left(x_{n}\right)-\psi(\hat{u})+\delta_{n} \mathcal{B} \psi(\hat{u})\right\|^{2}+\left(1-\eta_{n}\right)\left\|\psi\left(x_{n}\right)-\psi(\hat{u})\right\|^{2} \text {. }
\end{aligned}
$$

According to (3.3) and (3.8), we have

$$
\left\|\psi\left(x_{n+1}\right)-\psi(\hat{u})\right\| \leqslant \xi_{n}\left\|\psi\left(x_{n}\right)-\psi(\hat{u})\right\|+\left(1-\xi_{n}\right)\left\|v_{n}-\psi(\hat{u})\right\|
$$




$$
\begin{aligned}
\leqslant & \xi_{n}\left\|\psi\left(x_{n}\right)-\psi(\hat{u})\right\|+\left(1-\xi_{n}\right)\left[1-(1-\mu L / \delta) \eta_{n}\right]\left\|\psi\left(x_{n}\right)-\psi(\hat{u})\right\| \\
& +\left(1-\xi_{n}\right) \eta_{n}(\|\mu \vartheta(\hat{u})-\psi(\hat{u})\|+2 \xi\|\mathcal{B} \psi(\hat{u})\|) \\
= & {\left[1-(1-\mu L / \delta)\left(1-\xi_{n}\right) \eta_{n}\right]\left\|\psi\left(x_{n}\right)-\psi(\hat{u})\right\| } \\
& +(1-\mu \mathrm{L} / \delta)\left(1-\xi_{n}\right) \eta_{n} \frac{\|\mu \vartheta(\hat{u})-\psi(\hat{u})\|+2 \xi\|\mathcal{B} \psi(\hat{u})\|}{1-\mu L / \delta} .
\end{aligned}
$$

By induction

$$
\left\|\psi\left(x_{n}\right)-\psi(\hat{u})\right\| \leqslant \max \left\{\left\|\psi\left(x_{0}\right)-\psi(\hat{u})\right\|, \frac{\|\mu \vartheta(\hat{u})-\psi(\hat{u})\|+2 \xi\|\mathcal{B} \psi(\hat{u})\|}{1-\mu L / \delta}\right\} .
$$

It follows that

$$
\left\|x_{n}-\hat{u}\right\| \leqslant \frac{1}{\delta}\left\|\psi\left(x_{n}\right)-\psi(\hat{u})\right\| \leqslant \frac{1}{\delta} \max \left\{\left\|\psi\left(x_{0}\right)-\psi(\hat{u})\right\|, \frac{\|\mu \vartheta(\hat{u})-\psi(\hat{u})\|+2 \xi\|\mathcal{B} \psi(\hat{u})\|}{1-\mu L / \delta}\right\} .
$$

Hence, $\left\{\psi\left(x_{n}\right)\right\},\left\{x_{n}\right\},\left\{u_{n}\right\}$, and $\left\{v_{n}\right\}$ are all bounded.

From (3.3), we have

$$
\psi\left(x_{n+1}\right)-\psi\left(x_{n}\right)=\left(1-\xi_{n}\right)\left(v_{n}-\psi\left(x_{n}\right)\right) .
$$

Thus,

$$
\left\langle\psi\left(x_{n+1}\right)-\psi\left(x_{n}\right), \psi\left(x_{n}\right)-\psi(\hat{u})\right\rangle=\left(1-\xi_{n}\right)\left\langle v_{n}-\psi\left(x_{n}\right), \psi\left(x_{n}\right)-\psi(\hat{u})\right\rangle .
$$

Observe that

$$
\begin{aligned}
2\left\langle\psi\left(x_{n+1}\right)-\psi\left(x_{n}\right), \psi\left(x_{n}\right)-\psi(\hat{u})\right\rangle= & \left\|\psi\left(x_{n+1}\right)-\psi(\hat{u})\right\|^{2}-\left\|\psi\left(x_{n}\right)-\psi(\hat{u})\right\|^{2} \\
& -\left\|\psi\left(x_{n+1}\right)-\psi\left(x_{n}\right)\right\|^{2}
\end{aligned}
$$

and

$$
2\left\langle v_{n}-\psi\left(x_{n}\right), \psi\left(x_{n}\right)-\psi(\hat{u})\right\rangle=\left\|v_{n}-\psi(\hat{u})\right\|^{2}-\left\|\psi\left(x_{n}\right)-\psi(\hat{u})\right\|^{2}-\left\|v_{n}-\psi\left(x_{n}\right)\right\|^{2} .
$$

By virtue of (3.11), (3.12), and (3.13), we deduce

$$
\begin{aligned}
& \left\|\psi\left(x_{n+1}\right)-\psi(\hat{u})\right\|^{2}-\left\|\psi\left(x_{n}\right)-\psi(\hat{u})\right\|^{2}-\left\|\psi\left(x_{n+1}\right)-\psi\left(x_{n}\right)\right\|^{2} \\
& \quad=\left(1-\xi_{n}\right)\left[\left\|v_{n}-\psi(\hat{u})\right\|^{2}-\left\|\psi\left(x_{n}\right)-\psi(\hat{u})\right\|^{2}-\left\|v_{n}-\psi\left(x_{n}\right)\right\|^{2}\right] .
\end{aligned}
$$

Combining (3.9), (3.10) with (3.14), we have

$$
\begin{aligned}
\| & \psi\left(x_{n+1}\right)-\psi(\hat{u})\left\|^{2}-\right\| \psi\left(x_{n}\right)-\psi(\hat{u}) \|^{2} \\
= & \left(1-\xi_{n}\right)\left[\left\|v_{n}-\psi(\hat{u})\right\|^{2}-\left\|\psi\left(x_{n}\right)-\psi(\hat{u})\right\|^{2}-\left\|v_{n}-\psi\left(x_{n}\right)\right\|^{2}\right]+\left(1-\xi_{n}\right)^{2}\left\|v_{n}-\psi\left(x_{n}\right)\right\|^{2} \\
= & \left(1-\xi_{n}\right)\left[\left\|v_{n}-\psi(\hat{u})\right\|^{2}-\left\|\psi\left(x_{n}\right)-\psi(\hat{u})\right\|^{2}\right]-\xi_{n}\left(1-\xi_{n}\right)\left\|v_{n}-\psi\left(x_{n}\right)\right\|^{2} \\
\leqslant & \left(1-\xi_{n}\right)\left[\eta_{n}\left\|\mu \vartheta\left(x_{n}\right)-\psi(\hat{u})+\delta_{n} \mathcal{B} \psi(\hat{u})\right\|^{2}+\left(1-\eta_{n}\right)\left\|u_{n}-\psi(\hat{u})\right\|^{2}\right. \\
& \left.-\left\|\psi\left(x_{n}\right)-\psi(\hat{u})\right\|^{2}\right]-\xi_{n}\left(1-\xi_{n}\right)\left\|v_{n}-\psi\left(x_{n}\right)\right\|^{2} .
\end{aligned}
$$

Returning to (3.8), we get

$$
\begin{aligned}
\left\|v_{n}-\psi(\hat{u})\right\|^{2} \leqslant & {\left[1-(1-\mu \mathrm{L} / \delta) \eta_{n}\right]\left\|\psi\left(x_{n}\right)-\psi(\hat{u})\right\|^{2} } \\
& +(1-\mu L / \delta) \eta_{n}\left(\frac{\|\mu \vartheta(\hat{u})-\psi(\hat{u})\|+2 \xi\|\mathcal{B} \psi(\hat{u})\|}{(1-\mu \mathrm{L} / \delta)}\right)^{2} .
\end{aligned}
$$

Next, we consider two possible cases.

Case 1. Assume there exists some integer $m>0$ such that $\left\{\left\|\psi\left(x_{n}\right)-\psi(\hat{u})\right\|\right\}$ is decreasing for all $n \geqslant m$. 
In this case, we know that $\lim _{n \rightarrow \infty}\left\|\psi\left(x_{n}\right)-\psi(\hat{u})\right\|$ exists. From (3.15) and (3.16), we have

$$
\begin{aligned}
& \xi_{n}\left(1-\xi_{n}\right)\left\|v_{n}-\psi\left(x_{n}\right)\right\|^{2} \\
& \leqslant \\
& \leqslant \\
& \leqslant \\
& \quad\left\|\psi\left(x_{n}\right)-\psi\left(x_{n}\right)-\psi(\hat{u})\right\|^{2}-\left\|\psi\left(x_{n+1}\right)-\psi(\hat{u})\right\|^{2}+\left(1-\xi_{n}\right)\left[\left\|v_{n}-\psi(\hat{u})\right\|^{2}-\left\|\psi\left(x_{n}\right)-\psi(\hat{u})\right\|^{2}\right] \\
& \quad+(1-\mu L / \delta) \eta_{n}\left(\frac{\|\mu \vartheta(\hat{u})-\psi(\hat{u})\|+2 \xi\|\mathcal{B} \psi(\hat{u})\|}{(1-\mu L / \delta)}\right)^{2} \\
& \quad \rightarrow 0 .
\end{aligned}
$$

This together with (C2) implies that

$$
\lim _{n \rightarrow \infty}\left\|v_{n}-\psi\left(x_{n}\right)\right\|=0 .
$$

Furthermore, it follows from (3.10) that

$$
\lim _{n \rightarrow \infty}\left\|\psi\left(x_{n+1}\right)-\psi\left(x_{n}\right)\right\|=0 .
$$

By the convexity of the norm and (3.9), we have

$$
\begin{aligned}
\left\|\psi\left(x_{n+1}\right)-\psi(\hat{u})\right\|^{2}= & \left\|\xi_{n}\left(\psi\left(x_{n}\right)-\psi(\hat{u})\right)+\left(1-\xi_{n}\right)\left(v_{n}-\psi(\hat{u})\right)\right\|^{2} \\
\leqslant & \xi_{n}\left\|\psi\left(x_{n}\right)-\psi(\hat{u})\right\|^{2}+\left(1-\xi_{n}\right)\left\|v_{n}-\psi(\hat{u})\right\|^{2} \\
\leqslant & \xi_{n}\left\|\psi\left(x_{n}\right)-\psi(\hat{u})\right\|^{2}+\eta_{n}\left(1-\xi_{n}\right)\left\|\mu \vartheta\left(x_{n}\right)-\psi(\hat{u})+\delta_{n} \mathcal{B} \psi(\hat{u})\right\|^{2} \\
& +\left(1-\eta_{n}\right)\left(1-\xi_{n}\right)\left\|\psi\left(x_{n}\right)-\psi(\hat{u})\right\|^{2} \\
& +\left(1-\eta_{n}\right)\left(1-\xi_{n}\right) \delta_{n}\left(\delta_{n}-2 \xi\right)\left\|\mathcal{B} u_{n}-\mathcal{B} \psi(\hat{u})\right\|^{2} \\
& +\left(1-\eta_{n}\right)\left(1-\xi_{n}\right) \zeta_{n}\left(\zeta_{n}-2 \eta\right)\left\|\mathcal{A} x_{n}-\mathcal{A} \hat{u}\right\|^{2} \\
\leqslant & \left\|\psi\left(x_{n}\right)-\psi(\hat{u})\right\|^{2}+\eta_{n}\left(1-\xi_{n}\right)\left\|\mu \vartheta\left(x_{n}\right)-\psi(\hat{u})+\delta_{n} \mathcal{B} \psi(\hat{u})\right\|^{2} \\
& +\left(1-\eta_{n}\right)\left(1-\xi_{n}\right) \delta_{n}\left(\delta_{n}-2 \xi\right)\left\|\mathcal{B} u_{n}-\mathcal{B} \psi(\hat{u})\right\|^{2} \\
& +\left(1-\eta_{n}\right)\left(1-\xi_{n}\right) \zeta_{n}\left(\zeta_{n}-2 \eta\right)\left\|\mathcal{A} x_{n}-\mathcal{A} \hat{u}\right\|^{2} .
\end{aligned}
$$

Thus,

$$
\begin{aligned}
& \left(1-\eta_{n}\right)\left(1-\xi_{n}\right) \delta_{n}\left(2 \xi-\delta_{n}\right)\left\|\mathcal{B} u_{n}-\mathcal{B} \psi(\hat{u})\right\|^{2}+\left(1-\eta_{n}\right)\left(1-\xi_{n}\right) \zeta_{n}\left(2 \eta-\zeta_{n}\right)\left\|\mathcal{A} x_{n}-\mathcal{A} \hat{u}\right\|^{2} \\
& \leqslant \\
& \quad \leqslant \psi\left(x_{n}\right)-\psi(\hat{u})\left\|^{2}-\right\| \psi\left(x_{n+1}\right)-\psi(\hat{u})\left\|^{2}+\eta_{n}\left(1-\xi_{n}\right)\right\| \mu \vartheta\left(x_{n}\right)-\psi(\hat{u})+\delta_{n} \mathcal{B} \psi(\hat{u}) \|^{2} \\
& \left.\quad+\psi(\hat{u})\|+\| \psi\left(x_{n+1}\right)-\psi(\hat{u}) \|\right)\left\|\psi\left(x_{n+1}\right)-\psi\left(x_{n}\right)\right\| \\
& \quad+\eta_{n}\left(1-\xi_{n}\right)\left\|\mu \vartheta\left(x_{n}\right)-\psi(\hat{u})+\delta_{n} \mathcal{B} \psi(\hat{u})\right\|^{2} \\
& \quad \rightarrow 0 \quad \text { by (C1) and (3.17)). }
\end{aligned}
$$

Since $\liminf _{n \rightarrow \infty}\left(1-\xi_{n}\right)\left(1-\eta_{n}\right) \zeta_{n}\left(2 \eta-\zeta_{n}\right)>0$ and $\liminf _{n \rightarrow \infty}\left(1-\eta_{n}\right)\left(1-\xi_{n}\right) \delta_{n}\left(2 \xi-\delta_{n}\right)>0$, we obtain

$$
\lim _{n \rightarrow \infty}\left\|\mathcal{A} x_{n}-\mathcal{A} \hat{u}\right\|=0 \text { and } \lim _{n \rightarrow \infty}\left\|\mathcal{B} u_{n}-\mathcal{B} \psi(\hat{u})\right\|=0 .
$$

Set $z_{n}=\psi\left(x_{n}\right)-\zeta_{n} \mathcal{A} x_{n}-\left(\psi(\hat{u})-\zeta_{n} \mathcal{A} \hat{u}\right)$ for all $n$. Using the property (2.1) of projection, we get

$$
\begin{aligned}
\left\|u_{n}-\psi(\hat{u})\right\|^{2} & =\left\|\operatorname{proj}_{\mathcal{C}}\left[\psi\left(x_{n}\right)-\zeta_{n} \mathcal{A} x_{n}\right]-\operatorname{proj}_{\mathcal{C}}\left[\psi(\hat{u})-\zeta_{n} \mathcal{A} \hat{u}\right]\right\|^{2} \\
& \leqslant\left\langle z_{n}, u_{n}-\psi(\hat{u})\right\rangle \\
& =\frac{1}{2}\left\{\left\|z_{n}\right\|^{2}+\left\|u_{n}-\psi(\hat{u})\right\|^{2}-\left\|z_{n}-u_{n}+\psi(\hat{u})\right\|^{2}\right\} \\
& \leqslant \frac{1}{2}\left\{\left\|\psi\left(x_{n}\right)-\psi(\hat{u})\right\|^{2}+\left\|u_{n}-\psi(\hat{u})\right\|^{2}-\left\|\psi\left(x_{n}\right)-u_{n}-\zeta_{n}\left(\mathcal{A} x_{n}-\mathcal{A} \hat{u}\right)\right\|^{2}\right\}
\end{aligned}
$$




$$
\begin{aligned}
= & \frac{1}{2}\left\{\left\|\psi\left(x_{n}\right)-\psi(\hat{u})\right\|^{2}+\left\|u_{n}-\psi(\hat{u})\right\|^{2}-\left\|\psi\left(x_{n}\right)-u_{n}\right\|^{2}\right. \\
& \left.-\zeta_{n}^{2}\left\|\mathcal{A} x_{n}-\mathcal{A} \hat{u}\right\|^{2}+2 \zeta_{n}\left\langle\psi\left(x_{n}\right)-u_{n}, \mathcal{A} x_{n}-\mathcal{A} \hat{u}\right\rangle\right\} .
\end{aligned}
$$

It follows that

$$
\left\|u_{n}-\psi(\hat{u})\right\|^{2} \leqslant\left\|\psi\left(x_{n}\right)-\psi(\hat{u})\right\|^{2}-\left\|\psi\left(x_{n}\right)-u_{n}\right\|^{2}+2 \zeta_{n}\left\|\psi\left(x_{n}\right)-u_{n}\right\|\left\|\mathcal{A} x_{n}-\mathcal{A} \hat{u}\right\| .
$$

Set $z_{n}^{\prime}=u_{n}-\delta_{n} \mathcal{B} u_{n}-\left(\psi(\hat{u})-\delta_{n} \mathcal{B} \psi(\hat{u})\right)$ for all $n$. We also have

$$
\begin{aligned}
\left\|v_{n}-\psi(\hat{u})\right\|^{2}= & \left\|\operatorname{proj}_{\mathcal{e}}\left[\eta_{n} \mu \vartheta\left(x_{n}\right)+\left(1-\eta_{n}\right)\left(u_{n}-\delta_{n} \mathcal{B} u_{n}\right)\right]-\operatorname{proj}_{\mathcal{e}}\left[\psi(\hat{u})-\delta_{n} \mathcal{B} \psi(\hat{u})\right]\right\|^{2} \\
\leqslant & \left\langle\eta_{n}\left(\mu \vartheta\left(x_{n}\right)-\psi(\hat{u})+\delta_{n} \mathcal{B} \psi(\hat{u})\right)+\left(1-\eta_{n}\right) z_{n}^{\prime}, v_{n}-\psi(\hat{u})\right\rangle \\
= & \frac{1}{2}\left\{\left\|\eta_{n}\left(\mu \vartheta\left(x_{n}\right)-\psi(\hat{u})+\delta_{n} \mathcal{B} \psi(\hat{u})\right)+\left(1-\eta_{n}\right) z_{n}^{\prime}\right\|^{2}+\left\|v_{n}-\psi(\hat{u})\right\|^{2}\right. \\
& \left.-\left\|\eta_{n}\left(\mu \vartheta\left(x_{n}\right)-\psi(\hat{u})+\delta_{n} \mathcal{B} \psi(\hat{u})\right)+\left(1-\eta_{n}\right) z_{n}^{\prime}-v_{n}+\psi(\hat{u})\right\|^{2}\right\} \\
\leqslant & \frac{1}{2}\left\{\eta_{n}\left\|\mu \vartheta\left(x_{n}\right)-\psi(\hat{u})+\delta_{n} \mathcal{B} \psi(\hat{u})\right\|^{2}+\left(1-\eta_{n}\right)\left\|u_{n}-\psi(\hat{u})\right\|^{2}\right. \\
& +\left\|v_{n}-\psi(\hat{u})\right\|^{2}-\| \eta_{n}\left(\mu \vartheta\left(x_{n}\right)-\psi(\hat{u})+\delta_{n} \mathcal{B} \psi(\hat{u})-z_{n}^{\prime}\right) \\
& \left.+u_{n}-v_{n}-\delta_{n}\left(\mathcal{B} u_{n}-\mathcal{B} \psi(\hat{u})\right) \|^{2}\right\} \\
= & \frac{1}{2}\left\{\eta_{n}\left\|\mu \vartheta\left(x_{n}\right)-\psi(\hat{u})+\delta_{n} \mathcal{B} \psi(\hat{u})\right\|^{2}+\left(1-\eta_{n}\right)\left\|u_{n}-\psi(\hat{u})\right\|^{2}\right. \\
& +\left\|v_{n}-\psi(\hat{u})\right\|^{2}-\left\|u_{n}-v_{n}\right\|^{2}-\eta_{n}^{2}\left\|\mu \vartheta\left(x_{n}\right)-\psi(\hat{u})+\delta_{n} \mathcal{B} \psi(\hat{u})-z_{n}^{\prime}\right\|^{2} \\
& +2 \delta_{n} \eta_{n}\left\langle\mathcal{B} u_{n}-\mathcal{B} \psi(\hat{u}), \mu \vartheta\left(x_{n}\right)-\psi(\hat{u})+\delta_{n} \mathcal{B} \psi(\hat{u})-z_{n}^{\prime}\right\rangle \\
& -\delta_{n}^{2}\left\|\mathcal{B} u_{n}-\mathcal{B} \psi(\hat{u})\right\|^{2}+2 \delta_{n}\left\langle u_{n}-v_{n}, \mathcal{B} u_{n}-\mathcal{B} \psi(\hat{u})\right\rangle \\
& \left.-2 \eta_{n}\left\langle u_{n}-v_{n}, \mu \vartheta\left(x_{n}\right)-\psi(\hat{u})+\delta_{n} \mathcal{B} \psi(\hat{u})-z_{n}^{\prime}\right\rangle\right\} .
\end{aligned}
$$

It follows that

$$
\begin{aligned}
\left\|v_{n}-\psi(\hat{u})\right\|^{2} \leqslant & \eta_{n}\left\|\mu \vartheta\left(x_{n}\right)-\psi(\hat{u})+\delta_{n} \mathcal{B} \psi(\hat{u})\right\|^{2}+\left(1-\eta_{n}\right)\left\|u_{n}-\psi(\hat{u})\right\|^{2} \\
& +2 \delta_{n} \eta_{n}\left\|\mathcal{B} u_{n}-\mathcal{B} \psi(\hat{u})\right\|\left\|\mu \vartheta\left(x_{n}\right)-\psi(\hat{u})+\delta_{n} \mathcal{B} \psi(\hat{u})-z_{n}^{\prime}\right\| \\
& -\left\|u_{n}-v_{n}\right\|^{2}+2 \delta_{n}\left\|u_{n}-v_{n}\right\|\left\|\mathcal{B} u_{n}-\mathcal{B} \psi(\hat{u})\right\| \\
& +2 \eta_{n}\left\|u_{n}-v_{n}\right\|\left\|\mu \vartheta\left(x_{n}\right)-\psi(\hat{u})+\delta_{n} \mathcal{B} \psi(\hat{u})-z_{n}^{\prime}\right\| .
\end{aligned}
$$

In the light of (3.18), (3.20), and (3.21), we derive

$$
\begin{aligned}
& \left\|\psi\left(x_{n+1}\right)-\psi(\hat{u})\right\|^{2} \\
& \leqslant \\
& \leqslant \\
& \leqslant \\
& \leqslant \\
& \quad\left\|\psi\left(x_{n}\right)-\psi(\hat{u})\right\|^{2}+\eta_{n}\left\|\mu \vartheta\left(x_{n}\right)-\psi(\hat{u})\right\|^{2}+\left(1-\xi_{n}\right)\left\|v_{n}-\psi(\hat{u})\right\|^{2} \\
& \quad-\left(1-\xi_{n}\right)\left\|u_{n}-v_{n}\right\|^{2}+2 \zeta_{n}\left\|\psi\left(x_{n}\right)-u_{n}\right\|\left\|\mathcal{A} x_{n}-\mathcal{A} \hat{u}\right\|+2 \delta_{n}\left\|u_{n}-v_{n}\right\|\left\|\mathcal{B} u_{n}-\mathcal{B} \psi(\hat{u})\right\| \\
& \quad+2 \delta_{n} \eta_{n}\left\|\mathcal{B} u_{n}-\mathcal{B} \psi(\hat{u})\right\|\left\|\mu \vartheta\left(x_{n}\right)-\psi(\hat{u})+\delta_{n} \mathcal{B} \psi(\hat{u})-z_{n}^{\prime}\right\| \\
& \quad+2 \eta_{n}\left\|u_{n}-v_{n}\right\|\left\|\mu \vartheta\left(x_{n}\right)-\psi(\hat{u})+\delta_{n} \mathcal{B} \psi(\hat{u})-z_{n}^{\prime}\right\| .
\end{aligned}
$$

Then,

$$
\begin{aligned}
(1- & \left.\eta_{n}\right)\left\|\psi\left(x_{n}\right)-u_{n}\right\|^{2}+\left(1-\xi_{n}\right)\left\|u_{n}-v_{n}\right\|^{2} \\
\leqslant & \left(\left\|\psi\left(x_{n}\right)-\psi(\hat{u})\right\|+\left\|\psi\left(x_{n+1}\right)-\psi(\hat{u})\right\|\right)\left\|\psi\left(x_{n+1}\right)-\psi\left(x_{n}\right)\right\| \\
& +2 \zeta_{n}\left\|\psi\left(x_{n}\right)-u_{n}\right\|\left\|\mathcal{A} x_{n}-\mathcal{A} \hat{u}\right\|+2 \delta_{n}\left\|u_{n}-v_{n}\right\|\left\|\mathcal{B} u_{n}-\mathcal{B} \psi(\hat{u})\right\| \\
& +2 \delta_{n} \eta_{n}\left\|\mathcal{B} u_{n}-\mathcal{B} \psi(\hat{u})\right\|\left\|\mu \vartheta\left(x_{n}\right)-\psi(\hat{u})+\delta_{n} \mathcal{B} \psi(\hat{u})-z_{n}^{\prime}\right\|
\end{aligned}
$$




$$
\begin{aligned}
& +2 \eta_{n}\left\|u_{n}-v_{n}\right\|\left\|\mu \vartheta\left(x_{n}\right)-\psi(\hat{u})+\delta_{n} \mathcal{B} \psi(\hat{u})-z_{n}^{\prime}\right\| \\
& +\eta_{n}\left\|\mu \vartheta\left(x_{n}\right)-\psi(\hat{u})+\delta_{n} \mathcal{B} \psi(\hat{u})\right\|^{2} \\
& \rightarrow 0 \quad \text { (by (C1), (3.17), and (3.19)). }
\end{aligned}
$$

Therefore,

$$
\lim _{n \rightarrow \infty}\left\|\psi\left(x_{n}\right)-u_{n}\right\|=0 \text { and } \quad \lim _{n \rightarrow \infty}\left\|u_{n}-v_{n}\right\|=0 .
$$

Now, we prove $\varlimsup_{n \rightarrow \infty}\left\langle\mu \vartheta(\hat{u})-\psi(\hat{u}), v_{n}-\psi(\hat{u})\right\rangle \leqslant 0$. Choose a subsequence $\left\{v_{n_{i}}\right\}$ of $\left\{v_{n}\right\}$ such that

$$
\varlimsup_{n \rightarrow \infty}\left\langle\mu \vartheta(\hat{u})-\psi(\hat{u}), v_{n}-\psi(\hat{u})\right\rangle=\lim _{i \rightarrow \infty}\left\langle\mu \vartheta(\hat{u})-\psi(\hat{u}), \nu_{n_{i}}-\psi(\hat{u})\right\rangle .
$$

Since $\left\{x_{n_{i}}\right\}$ is bounded, there exists a subsequence $\left\{x_{n_{i_{j}}}\right\}$ of $\left\{x_{n_{i}}\right\}$ which converges weakly to some point $z \in \mathcal{C}$. Without loss of generality, we may assume that $x_{n_{i}} \rightarrow z$. This implies that $\psi\left(x_{n_{i}}\right) \rightarrow \psi(z)$ due to the weak continuity of $\psi$. Thus, $v_{n_{i}} \rightarrow \psi(z)$ by (3.22). Next, we show $z \in \operatorname{VI}(\mathcal{A}, \psi, \mathcal{C})$. Set

$$
\mathrm{Ru}= \begin{cases}\mathcal{A u}+\mathrm{N}_{\mathcal{C}}(u), & \mathrm{u} \in \mathcal{C}, \\ \emptyset, & \mathrm{u} \notin \mathcal{C} .\end{cases}
$$

By [35], we know that $R$ is maximal $\psi$-monotone. Let $(u, w) \in G(R)$. Since $w-B u \in N_{\mathcal{C}}(u)$ and $x_{n} \in \mathcal{C}$, we have $\left\langle\psi(u)-\psi\left(x_{n}\right), w-\mathcal{A} u\right\rangle \geqslant 0$. Noting that $u_{n}=\operatorname{proj}_{\mathfrak{e}}\left[\psi\left(x_{n}\right)-\zeta_{n} \mathcal{A} x_{n}\right]$, we get

$$
\left\langle\psi(u)-u_{n}, u_{n}-\left(\psi\left(x_{n}\right)-\zeta_{n} \mathcal{A} x_{n}\right)\right\rangle \geqslant 0 .
$$

It follows that

$$
\left\langle\psi(u)-u_{n}, \frac{u_{n}-\psi\left(x_{n}\right)}{\zeta_{n}}+\mathcal{A} x_{n}\right\rangle \geqslant 0
$$

Then,

$$
\begin{aligned}
\left\langle\psi(u)-\psi\left(x_{n_{i}}\right), w\right\rangle \geqslant & \left\langle\psi(u)-\psi\left(x_{n_{i}}\right), \mathcal{A v}\right\rangle \\
\geqslant & \left\langle\psi(u)-\psi\left(x_{n_{i}}\right), \mathcal{A u}\right\rangle-\left\langle\psi(u)-u_{n_{i}}, \frac{u_{n_{i}}-\psi\left(x_{n_{i}}\right)}{\zeta_{n_{i}}}\right\rangle-\left\langle\psi(u)-u_{n_{i}}, \mathcal{A} x_{n_{i}}\right\rangle \\
= & \left\langle\psi(u)-\psi\left(x_{n_{i}}\right), \mathcal{A u}-\mathcal{A} x_{n_{i}}\right\rangle+\left\langle\psi(u)-\psi\left(x_{n_{i}}\right), \mathcal{A} x_{n_{i}}\right\rangle \\
& -\left\langle\psi(u)-u_{n_{i}}, \frac{u_{n_{i}}-\psi\left(x_{n_{i}}\right)}{\zeta_{n_{i}}}\right\rangle-\left\langle\psi(u)-u_{n_{i}}, \mathcal{A} x_{n_{i}}\right\rangle \\
\geqslant & -\left\langle\psi(u)-u_{n_{i}}, \frac{u_{n_{i}}-\psi\left(x_{n_{i}}\right)}{\zeta_{n_{i}}}\right\rangle-\left\langle\psi\left(x_{n_{i}}\right)-u_{n_{i}}, \mathcal{A} x_{n_{i}}\right\rangle .
\end{aligned}
$$

Since $\left\|\psi\left(x_{n_{i}}\right)-u_{n_{i}}\right\| \rightarrow 0$ and $\psi\left(x_{n_{i}}\right) \rightarrow \psi(z)$, we deduce that $\langle\psi(u)-\psi(z), w\rangle \geqslant 0$ by taking $i \rightarrow \infty$ in (3.24). Thus, $z \in \mathrm{R}^{-1} 0$ by the maximal $\psi$-monotonicity of $\mathrm{R}$. Hence, $z \in \operatorname{VI}(\mathcal{A}, \psi, \mathcal{C})$.

Next, we need to prove $\psi(z) \in \mathrm{VI}(\mathcal{B}, \mathcal{C})$. Set

$$
\mathrm{R}^{\prime} v= \begin{cases}\mathcal{B} v+\mathrm{N}_{\mathcal{C}}(v), & v \in \mathcal{C}, \\ \emptyset, & v \notin \mathcal{C} .\end{cases}
$$

By [21], we know that $R^{\prime}$ is maximal monotone. Let $(v, w) \in G\left(R^{\prime}\right)$. Since $w-\mathcal{B} v \in N_{\mathcal{C}}(v)$ and $v_{n} \in \mathcal{C}$, we have $\left\langle v-v_{n}, w-\mathcal{B} v\right\rangle \geqslant 0$. Noting that $v_{n}=\operatorname{proj}_{\mathcal{C}}\left[\eta_{n} \mu \vartheta\left(x_{n}\right)+\left(1-\eta_{n}\right)\left(u_{n}-\delta_{n} \mathcal{B} u_{n}\right)\right]$, we get

$$
\left\langle v-v_{n}, v_{n}-\left[\eta_{n} \mu \vartheta\left(x_{n}\right)+\left(1-\eta_{n}\right)\left(u_{n}-\delta_{n} \mathcal{B} u_{n}\right)\right]\right\rangle \geqslant 0 .
$$

It follows that

$$
\left\langle v-v_{n}, \frac{v_{n}-u_{n}}{\delta_{n}}+\mathcal{B} u_{n}-\frac{\eta_{n}}{\delta_{n}}\left(\mu \vartheta\left(x_{n}\right)-u_{n}+\delta_{n} \mathcal{B} u_{n}\right)\right\rangle \geqslant 0
$$


Then,

$$
\begin{aligned}
\left\langle v-v_{n_{i}}, w\right\rangle \geqslant & \left\langle v-v_{n_{i}}, \mathcal{B} v\right\rangle \\
\geqslant & \left\langle v-v_{n_{i}}, \mathcal{B} v\right\rangle-\left\langle v-v_{n_{i}}, \frac{v_{n_{i}}-u_{n_{i}}}{\delta_{n_{i}}}\right\rangle-\left\langle v-v_{n_{i}}, \mathcal{B} u_{n_{i}}\right\rangle \\
& +\frac{\eta_{n_{i}}}{\delta_{n_{i}}}\left\langle v-v_{n_{i}}, \mu \vartheta\left(x_{n_{i}}\right)-u_{n_{i}}+\delta_{n_{i}} \mathcal{B} u_{n_{i}}\right\rangle \\
= & \left\langle v-v_{n_{i}}, \mathcal{B} v-\mathcal{B} v_{n_{i}}\right\rangle+\left\langle v-v_{n_{i}}, \mathcal{B} v_{n_{i}}\right\rangle-\left\langle v-v_{n_{i}}, \frac{v_{n_{i}}-u_{n_{i}}}{\delta_{n_{i}}}\right\rangle \\
& -\left\langle v-v_{n_{i}}, \mathcal{B} u_{n_{i}}\right\rangle+\frac{\eta_{n_{i}}}{\delta_{n_{i}}}\left\langle v-v_{n_{i}}, \mu \vartheta\left(x_{n_{i}}\right)-u_{n_{i}}+\delta_{n_{i}} \mathcal{B} u_{n_{i}}\right\rangle \\
\geqslant & -\left\langle v-v_{n_{i}}, \frac{v_{n_{i}}-u_{n_{i}}}{\delta_{n_{i}}}\right\rangle-\left\langle v-v_{n_{i}}, \mathcal{B} u_{n_{i}}-\mathcal{B} v_{n_{i}}\right\rangle \\
& +\frac{\eta_{n_{i}}}{\delta_{n_{i}}}\left\langle v-v_{n_{i}}, \mu \vartheta\left(x_{n_{i}}\right)-u_{n_{i}}+\delta_{n_{i}} \mathcal{B} u_{n_{i}}\right\rangle .
\end{aligned}
$$

Since $\left\|v_{n_{i}}-u_{n_{i}}\right\| \rightarrow 0$ and $v_{n_{i}} \rightarrow \psi(z)$, we deduce that $\langle v-\psi(z), w\rangle \geqslant 0$ by taking $i \rightarrow \infty$ in (3.25). Thus, $\psi(z) \in \mathrm{R}^{\prime-1} 0$ by the maximal monotonicity of $\mathrm{R}^{\prime}$. Hence, $\psi(z) \in \mathrm{VI}(\mathcal{B}, \mathcal{C})$. Therefore, $z \in \Lambda$.

From (3.23), we obtain

$$
\begin{aligned}
\varlimsup_{n \rightarrow \infty}\left\langle\mu \vartheta(\hat{u})-\psi(\hat{u}), v_{n}-\psi(\hat{u})\right\rangle & =\lim _{i \rightarrow \infty}\left\langle\mu \vartheta(\hat{u})-\psi(\hat{u}), \psi\left(x_{n_{i}}\right)-\psi(\hat{u})\right\rangle \\
& =\langle\mu \vartheta(\hat{u})-\psi(\hat{u}), \psi(z)-\psi(\hat{u})\rangle \leqslant 0 .
\end{aligned}
$$

Note that

$$
\begin{aligned}
\left\|v_{n}-\psi(\hat{u})\right\|^{2}= & \left\|\operatorname{proj}_{\mathcal{e}}\left[\eta_{n} \mu \vartheta\left(x_{n}\right)+\left(1-\eta_{n}\right)\left(u_{n}-\delta_{n} \mathcal{B} u_{n}\right)\right]-\operatorname{proj}_{\mathcal{C}}\left[\psi(\hat{u})-\left(1-\eta_{n}\right) \delta_{n} \mathcal{B} \psi(\hat{u})\right]\right\|^{2} \\
\leqslant & \left\langle\eta_{n}\left(\mu \vartheta\left(x_{n}\right)-\psi(\hat{u})\right)+\left(1-\eta_{n}\right) z_{n}^{\prime}, v_{n}-\psi(\hat{u})\right\rangle \\
\leqslant & \eta_{n} \mu\left\langle\vartheta\left(x_{n}\right)-\vartheta(\hat{u}), v_{n}-\psi(\hat{u})\right\rangle+\eta_{n}\left\langle\mu \vartheta(\hat{u})-\psi(\hat{u}), v_{n}-\psi(\hat{u})\right\rangle \\
& \left.+\left(1-\eta_{n}\right) \| u_{n}-\delta_{n} \mathcal{B} u_{n}-\psi(\hat{u})-\delta_{n} \mathcal{B} \psi(\hat{u})\right)\|\| v_{n}-\psi(\hat{u}) \| \\
\leqslant & \eta_{n} L \mu\left\|x_{n}-\hat{u}\right\|\left\|v_{n}-\psi(\hat{u})\right\|+\eta_{n}\left\langle\mu \vartheta(\hat{u})-\psi(\hat{u}), v_{n}-\psi(\hat{u})\right\rangle \\
& +\left(1-\eta_{n}\right)\left\|u_{n}-\psi(\hat{u})\right\|\left\|v_{n}-\psi(\hat{u})\right\| \\
\leqslant & \eta_{n}(\mu L / \delta)\left\|\psi\left(x_{n}\right)-\psi(\hat{u})\right\|\left\|v_{n}-\psi(\hat{u})\right\|+\eta_{n}\left\langle\mu \vartheta(\hat{u})-\psi(\hat{u}), v_{n}-\psi(\hat{u})\right\rangle \\
& +\left(1-\eta_{n}\right)\left\|\psi\left(x_{n}\right)-\psi(\hat{u})\right\|\left\|v_{n}-\psi(\hat{u})\right\| \\
= & {\left[1-(1-L \mu / \delta) \eta_{n}\right]\left\|\psi\left(x_{n}\right)-\psi(\hat{u})\right\|\left\|v_{n}-\psi(\hat{u})\right\|+\eta_{n}\left\langle\mu \vartheta(\hat{u})-\psi(\hat{u}), v_{n}-\psi(\hat{u})\right\rangle } \\
\leqslant & \frac{1-(1-L \mu / \delta) \eta_{n}}{2}\left\|\psi\left(x_{n}\right)-\psi(\hat{u})\right\|^{2}+\frac{1}{2}\left\|v_{n}-\psi(\hat{u})\right\|^{2}+\eta_{n}\left\langle\mu \vartheta(\hat{u})-\psi(\hat{u}), v_{n}-\psi(\hat{u})\right\rangle .
\end{aligned}
$$

It follows that

$$
\left\|v_{n}-\psi(\hat{u})\right\|^{2} \leqslant\left[1-(1-L \mu / \delta) \eta_{n}\right]\left\|\psi\left(x_{n}\right)-\psi(\hat{u})\right\|^{2}+2 \eta_{n}\left\langle\mu \vartheta(\hat{u})-\psi(\hat{u}), v_{n}-\psi(\hat{u})\right\rangle .
$$

Therefore,

$$
\begin{aligned}
\left\|\psi\left(x_{n+1}\right)-\psi(\hat{u})\right\|^{2} \leqslant & \xi_{n}\left\|\psi\left(x_{n}\right)-\psi(\hat{u})\right\|^{2}+\left(1-\xi_{n}\right)\left\|v_{n}-\psi(\hat{u})\right\|^{2} \\
\leqslant & \xi_{n}\left\|\psi\left(x_{n}\right)-\psi(\hat{u})\right\|^{2}+\left(1-\xi_{n}\right)\left[1-(1-\mu L / \delta) \eta_{n}\right]\left\|\psi\left(x_{n}\right)-\psi(\hat{u})\right\|^{2} \\
& +2\left(1-\xi_{n}\right) \eta_{n}\left\langle\mu \vartheta(\hat{u})-\psi(\hat{u}), v_{n}-\psi(\hat{u})\right\rangle \\
= & {\left[1-(1-\mu L / \delta)\left(1-\xi_{n}\right) \eta_{n}\right]\left\|\psi\left(x_{n}\right)-\psi(\hat{u})\right\|^{2} } \\
& +2\left(1-\xi_{n}\right) \eta_{n}\left\langle\mu \vartheta(\hat{u})-\psi(\hat{u}), v_{n}-\psi(\hat{u})\right\rangle \\
= & {\left[1-(1-\mu L / \delta)\left(1-\xi_{n}\right) \eta_{n}\right]\left\|\psi\left(x_{n}\right)-\psi(\hat{u})\right\|^{2} }
\end{aligned}
$$




$$
+(1-\mu \mathrm{L} / \delta)\left(1-\xi_{n}\right) \eta_{n}\left(\frac{2}{1-\mu \mathrm{L} / \delta}\left\langle\mu \vartheta(\hat{\mathrm{u}})-\psi(\hat{\mathrm{u}}), \nu_{\mathrm{n}}-\psi(\hat{\mathrm{u}})\right\rangle\right) .
$$

We can apply Lemma 2.6 to (3.27) to conclude that $\psi\left(x_{n}\right) \rightarrow \psi(\hat{u})$ and $x_{n} \rightarrow \hat{u}$.

Case 2. Assume there exists an integer $n_{0}$ such that $\left\|\psi\left(x_{n_{0}}\right)-\psi(\hat{u})\right\| \leqslant\left\|\psi\left(x_{n_{0}+1}\right)-\psi(\hat{u})\right\|$. At this case, we set $\omega_{n}=\left\{\left\|\psi\left(x_{n}\right)-\psi(\hat{u})\right\|\right\}$. Then, we have $\omega_{n_{0}} \leqslant \omega_{n_{0}+1}$. Define an integer sequence $\left\{\tau_{n}\right\}$ for all $n \geqslant n_{0}$ as follows:

$$
\tau(n)=\max \left\{l \in \mathbb{N} \mid n_{0} \leqslant l \leqslant n, \omega_{l} \leqslant \omega_{l+1}\right\} .
$$

It is clear that $\tau(n)$ is a non-decreasing sequence satisfying

$$
\lim _{n \rightarrow \infty} \tau(n)=\infty
$$

and

$$
\omega_{\tau(n)} \leqslant \omega_{\tau(n)+1},
$$

for all $n \geqslant n_{0}$.

By the similar argument as that of (3.26) and (3.27), we can prove that

$$
\varlimsup_{n \rightarrow \infty}\left\langle\mu \vartheta(\hat{u})-\psi(\hat{u}), \nu_{\tau(n)}-\psi(\hat{u})\right\rangle \leqslant 0
$$

and

$$
\begin{aligned}
\omega_{\tau(n)+1}^{2} \leqslant & {\left[1-(1-\mu L / \delta)\left(1-\xi_{\tau(n)}\right) \eta_{\tau(n)}\right] \omega_{\tau(n)}^{2} } \\
& +(1-\mu L / \delta)\left(1-\xi_{\tau(n)}\right) \eta_{\tau(n)}\left(\frac{2}{1-\mu L / \delta}\left\langle\mu \vartheta(\hat{u})-\psi(\hat{u}), \nu_{\tau(n)}-\psi(\hat{u})\right\rangle\right) .
\end{aligned}
$$

Since $\omega_{\tau(n)} \leqslant \omega_{\tau(n)+1}$, we have from (3.29) that

$$
\omega_{\tau(n)}^{2} \leqslant \frac{2}{1-\mu L / \delta}\left\langle\mu \vartheta(\hat{u})-\psi(\hat{u}), \nu_{\tau(n)}-\psi(\hat{u})\right\rangle .
$$

Combining (3.28) with (3.30), we have

$$
\varlimsup_{n \rightarrow \infty} \omega_{\tau(n)} \leqslant 0,
$$

and hence

$$
\lim _{n \rightarrow \infty} \omega_{\tau(n)}=0
$$

From (3.28) and (3.29), we also obtain

$$
\varlimsup_{n \rightarrow \infty} \omega_{\tau(n)+1} \leqslant \overline{\lim }_{n \rightarrow \infty} \omega_{\tau(n)} .
$$

This together with (3.31) implies that

$$
\lim _{n \rightarrow \infty} \omega_{\tau(n)+1}=0
$$

Applying Lemma 2.7 we get

$$
0 \leqslant \omega_{n} \leqslant \max \left\{\omega_{\tau(n)}, \omega_{\tau(n)+1}\right\} .
$$

Therefore, $\omega_{n} \rightarrow 0$. That is, $x_{n} \rightarrow \hat{u}$. This completes the proof.

Algorithm 3.7. For given initial value $x_{0} \in \mathcal{C}$, define the sequence $\left\{x_{n}\right\}$ by the following form

$$
\left\{\begin{array}{l}
u_{n}=\operatorname{proj}_{\mathcal{C}}\left[x_{n}-\zeta_{n} \mathcal{A} x_{n}\right], \\
v_{n}=\operatorname{proj}_{\mathcal{C}}\left[\eta_{n} \mu \vartheta\left(x_{n}\right)+\left(1-\eta_{n}\right)\left(u_{n}-\delta_{n} \mathcal{B} u_{n}\right)\right], \\
x_{n+1}=\xi_{n} x_{n}+\left(1-\xi_{n}\right) v_{n}, n \geqslant 0,
\end{array}\right.
$$

where $\vartheta: \mathcal{C} \rightarrow \mathcal{H}$ is an L-Lipschitz continuous mapping, $\left\{\eta_{n}\right\},\left\{\xi_{n}\right\}$, and $\left\{\delta_{n}\right\}$ are three real number sequences in $[0,1],\left\{\zeta_{n}\right\}$ is a real number sequence in $(0, \infty)$, and $0<\mu<1 / \mathrm{L}$ is a constant. 
Corollary 3.8. Assume the following conditions are satisfied:

(C1): $\lim _{n \rightarrow \infty} \eta_{n}=0$ and $\sum_{n} \eta_{n}=\infty$;

(C2): $0<\underline{\lim }_{n \rightarrow \infty} \xi_{n} \leqslant \varlimsup_{n \rightarrow \infty} \xi_{n}<1$;

(C3): $0<\underline{\lim }_{n \rightarrow \infty} \zeta_{n} \leqslant \varlimsup_{n \rightarrow \infty} \zeta_{n}<2 \eta$ and $0<\underline{\lim }_{n \rightarrow \infty} \delta_{n} \leqslant \varlimsup_{n \rightarrow \infty} \delta_{n}<2 \xi$.

Then the sequence $\left\{x_{n}\right\}$ generated by (3.32) converges strongly to $\hat{u} \in V I(A, C) \cap V I(B, C)$ which solves the following variational inequality

$$
\langle\mu \vartheta(\hat{u})-\hat{u}, \hat{v}-\hat{u}\rangle \leqslant 0, \quad \forall \hat{v} \in \operatorname{VI}(A, C) \cap \operatorname{VI}(B, C) .
$$

\section{References}

[1] M. Aslam Noor, Some developments in general variational inequalities, Appl. Math. Comput., 152 (2004), 199-277. 1

[2] C. Byrne, A unified treatment of some iterative algorithms in signal processing and image reconstruction, Inverse Problems, 20 (2004), 103-120. 1

[3] L.-C. Ceng, Q. H. Ansari, J.-C. Yao, An extragradient method for solving split feasibility and fixed point problems, Comput. Math. Appl., 64 (2012), 633-642.

[4] L.-C. Ceng, Q. H. Ansari, J.-C. Yao, Relaxed extragradient methods for finding minimum-norm solutions of the split feasibility problem, Nonlinear Anal., 75 (2012), 2116-2125. 1

[5] L.-C. Ceng, M. Teboulle, J.-C. Yao, Weak convergence of an iterative method for pseudomonotone variational inequalities and fixed-point problems, J. Optim. Theory Appl., 146 (2010), 19-31. 1

[6] L.-C. Ceng, J.-C. Yao, Strong convergence theorem by an extragradient method for fixed point problems and variational inequality problems, Taiwanese J. Math., 10 (2006), 1293-1303. 1

[7] Y. Censor, T. Bortfeld, B. Martin, A. Trofimov, A unified approach for inversion problems in intensity-modulated radiation therapy, Phys. Med. Biol., 51 (2006), 2353-2365. 1

[8] Y. Censor, T. Elfving, A multiprojection algorithm using Bregman projections in a product space, Numer. Algorithms, 8 (1994), 221-239.

[9] Y. Censor, T. Elfving, N. Kopf, T. Bortfeld, The multiple-sets split feasibility problem and its applications for inverse problems, Inverse Problems, 21 (2005), 2071-2084. 1

[10] Y. Censor, A. Gibali, S. Reich, Algorithms for the split variational inequality problem, Numer. Algorithms, 59 (2012), 301-323. ii

[11] Y. Censor, A. Segal, The split common fixed point problem for directed operators, J. Convex Anal., 16 (2009), 587-600. i

[12] F. Cianciaruso, G. Marino, L. Muglia, Y.-H. Yao, On a two-step algorithm for hierarchical fixed point problems and variational inequalities, J. Inequal. Appl., 2009 (2009), 13 pages. 1

[13] R. Glowinski, Numerical methods for nonlinear variational problems, Springer Series in Computational Physics, Springer-Verlag, New York, (1984). 1

[14] Z.-H. He, W.-S. Du, On hybrid split problem and its nonlinear algorithms, Fixed Point Theory Appl., 2013 (2013), 20 pages. i

[15] Z.-H. He, W.-S. Du, On split common solution problems: new nonlinear feasible algorithms, strong convergence results and their applications, Fixed Point Theory Appl., 2014 (2014), 16 pages. i

[16] A. N. Iusem, An iterative algorithm for the variational inequality problem, Mat. Apl. Comput., 13 (1994), 103-114. 1

[17] G. M. Korpelevič, An extragradient method for finding saddle points and for other problems, (Russian) Ékonom. i Mat. Metody, 12 (1976), 12 (1976), 747-756. 1

[18] P. E. Mainge, Approximation methods for common fixed points of nonexpansive mappings in Hilbert spaces, J. Math. Anal. Appl., 325 (2007), 469-479. 2.7

[19] X.-L. Qin, S. Y. Cho, Convergence analysis of a monotone projection algorithm in reflexive Banach spaces, Acta Math. Sci. Ser. B Engl. Ed., 37 (2017), 488-502. 1

[20] X.-L. Qin, J.-C. Yao, Weak convergence of a Mann-like algorithm for nonexpansive and accretive operators, J. Inequal. Appl., 2016 (2016), 9 pages. 1

[21] R.-T. Rockafellar, Monotone operators and the proximal point algorithm, SIAM J. Control Optimization, 14 (1976), 877-898. 3

[22] W. Takahashi, M. Toyoda, Weak convergence theorems for nonexpansive mappings and monotone mappings, J. Optim. Theory Appl., 118 (2003), 417-428. 2.5

[23] H.-K. Xu, Iterative algorithms for nonlinear operators, J. London Math. Soc., 2 (2002), 240-256. 2.6

[24] H.-K. Xu, A variable Krasnoselskiŭ-Mann algorithm and the multiple-set split feasibility problem, Inverse Problems, 22 (2006), 2021-2034. 1

[25] H.-K. Xu, Iterative methods for the split feasibility problem in infinite-dimensional Hilbert spaces, Inverse Problems, 26 (2010), 17 pages.

[26] Y.-H. Yao, R. P. Agarwal, M. Postolache, Y.-C. Liou, Algorithms with strong convergence for the split common solution of the feasibility problem and fixed point problem, Fixed Point Theory Appl., 183 (2014), 14 pages. 1 
[27] Y.-H. Yao, R.-D. Chen, H.-K. Xu, Schemes for finding minimum-norm solutions of variational inequalities, Nonlinear Anal., 72 (2010), 3447-3456. 1

[28] Y.-H. Yao, W. Jigang, Y.-C. Liou, Regularized methods for the split feasibility problem, Abstr. Appl. Anal., 2012 (2012), 13 pages. 1

[29] Y.-H. Yao, Y.-C. Liou, S. M. Kang, Approach to common elements of variational inequality problems and fixed point problems via a relaxed extragradient method, Comput. Math. Appl., 59 (2010), 3472-3480. 1

[30] Y.-H. Yao, Y.-C. Liou, J.-C. Yao, Split common fixed point problem for two quasi-pseudo-contractive operators and its algorithm construction, Fixed Point Theory Appl., 2015 (2015), 19 pages. i

[31] Y.-H. Yao, M. A. Noor, Y.-C. Liou, Strong convergence of a modified extragradient method to the minimum-norm solution of variational inequalities, Abstr. Appl. Anal., 2012 (2012), 9 pages. 1

[32] Y.-H. Yao, M. A. Noor, Y.-C. Liou, S. M. Kang, Iterative algorithms for general multivalued variational inequalities, Abstr. Appl. Anal., 2012 (2012), 10 pages. 1

[33] Y.-H. Yao, M. Postolache, Y.-C. Liou, Strong convergence of a self-adaptive method for the split feasibility problem, Fixed Point Theory Appl., 2013 (2013), 12 pages. 1

[34] H. Zegeye, N. Shahzad, Y.-H. Yao, Minimum-norm solution of variational inequality and fixed point problem in Banach spaces, Optimization, 64 (2015), 453-471. 1

[35] L. J. Zhang, J. M. Chen, Z. B. Hou, Viscosity approximation methods for nonexpansive mappings and generalized variational inequalities, (Chinese) Acta Math. Sinica (Chin. Ser.), 53 (2010), 691-698. 1, 3 